Trayectorias Universitarias, 7 (12), e056, 2021

ISSN 2469-0090 | https://doi.org/10.24215/24690090e056

https://revistas.unlp.edu.ar/TrayectoriasUniversitarias

Universidad Nacional de La Plata

La Plata | Buenos Aires | Argentina

\title{
Virtualización de una propuesta de alfabetización académica para estudiantes universitarios
}

\section{Virtualization of a course for academic literacy for university students}

\section{Carolina Clerici}

http://orcid.org/0000-0001-7261-3744

clericicarolina@hotmail.com

Universidad Nacional de Entre Ríos |

Argentina

\author{
María Consuelo Eckerdt \\ https://orcid.org/0000-0001-5503-5268 \\ consueloeckerdt@gmail.com \\ Universidad Nacional de Entre Ríos | \\ Argentina
}

\author{
Elisa Fernanda Naef \\ http://orcid.org/0000-0001-5892-831X \\ elisafnaef@hotmail.com \\ Universidad Nacional de Entre Ríos | \\ Argentina
}

\section{RESUMEN}

La alfabetización académica entendida como el ingreso a una nueva cultura discursiva es un desafío para quienes ingresan a la educación superior. En este contexto, los estudiantes se enfrentan con una nueva forma de leer y escribir que es necesario aprender. La inmersión en esta nueva cultura hace necesario que el estudiante se implique en actividades auténticas de comunicación, con actores reales de la comunidad universitaria. Se propuso la realización de un curso virtual de redacción académica y científica y Normas APA para los estudiantes de segundo año de la carrera presencial de Licenciatura en Nutrición de la Facultad de Bromatología (UNER), con la participación de estudiantes avanzados y docentes. Como virtualización se propuso e-learning asincrónico y autoaprendizaje con tutor. Se trabajó con lectura de documentos, resolución de tareas y foros temáticos como espacio de intercambio y trabajo colaborativo. La presente propuesta significó una oportunidad de interacción entre estudiantes y el resto de la comunidad universitaria en torno a una instancia de formación como es la alfabetización académica.

PALABRAS CLAVE

Alfabetización Académica, Virtualización, Estudiantes Universitarios. 


\section{KEY WORDS}

Academic Literacy, Virtualization,

University Students.

\begin{abstract}
Academic literacy understood as the entrance to a new discursive culture is a challenge for those who enter higher education. In this context, students face a new way of reading and writing that needs to be learned. Immersion in this new culture makes it necessary for the student to be involved in authentic communication activities, with real actors from the university community. A virtual course of academic and scientific writing and APA Standards was planned for second year students of the course in Nutrition from the School of Bromatology (UNER), with the participation of advanced students and teachers. The methodology was virtual education, asynchronous e-learning and self-learning with tutor. The activities involved reading documents, solving tasks and participating in forums as a space for exchange and collaborative work. This experience meant an opportunity for interaction between students and the rest of the university community within a course on academic literacy.
\end{abstract}




\section{1-INTRODUCCIÓN}

Alfabetización académica? ¿Un proceso tan básico en la educación superior? ¿Acaso una necesidad remedial de paliar lo que no han hecho los niveles escolares previos? ¿De nuevo alguien que propone un taller de lectura y escritura para los ingresantes? Con estas preguntas inició la Dra. Paula Carlino una ponencia sobre alfabetización académica hace más de una década. Esta autora sostiene que la fuerza de este concepto "radica en que pone de manifiesto que los modos de leer y escribir -de buscar, adquirir, elaborar y comunicar conocimientono son iguales en todos los ámbitos" (Carlino, 2003a, p.410).

Las prácticas discursivas propias de la vida académica con las que se encuentran los estudiantes al ingresar al nivel superior suponen una adaptación a nuevas formas de comprender, interpretar y organizar el conocimiento, "se trata de que al ingresar a la formación superior se les exige un cambio en su identidad como pensadores y analizadores de textos" (Carlino, 2003b, p. 17). Abundan los estudios que dan cuenta de que esos nuevos modos de leer y escribir se presentan como una dificultad para los estudiantes universitarios (Arnoux, Di Stefano y Pereira, 2002; Clerici, Monteverde y Fernández, 2015; Clerici, Truffer y Unamuzaga, 2009; González de la Torre, Jiménez Mora y Rosas, 2016).

La inmersión en la nueva cultura académica hace necesario que el estudiante se implique en actividades auténticas de comunicación, con actores reales de la comunidad universitaria. Los jóvenes aprenden cuando toman parte en actividades de su comunidad, involucrándose con otros jóvenes y adultos en procesos de colaboración (Rogoff, 1997) que les permiten prepararse para su ulterior participación en situaciones semejantes. En esta línea son reconocidos los postulados de Vigotsky (1987) quien sostiene que el aprendizaje se da como resultado de la interacción con miembros más expertos.

Desde el Curso de Ambientación a la Vida Universitaria y el taller de Lectura y Escritura con ingresantes a la Facultad de Bromatología de la Universidad Nacional de Entre Ríos (Clerici, 2016) se ha observado un preocupante aumento de las dificultades que tienen estos jóvenes para una comunicación efectiva, para el diálogo genuino, para la expresión de ideas con claridad. A esto se suma la tendencia a leer y estudiar de apuntes y resúmenes sin la consulta de textos académicos y científicos. Además, a diferencia de lo que podría creerse en la sociedad del conocimiento, no han desarrollado competencias para un uso 
eficiente de internet y en muchos casos no han trabajado en plataformas virtuales de aprendizaje.

Ante ese panorama, se propuso la realización de un curso virtual de redacción académica y científica y Normas APA para los estudiantes de segundo año de la Licenciatura en Nutrición de la Facultad de Bromatología de la Universidad Nacional de Entre Ríos, con la participación de estudiantes avanzados y docentes. Los principales objetivos del curso fueron ofrecer un espacio para conocer algunos géneros académicos y científicos, sus partes y rasgos distintivos; lograr un manejo adecuado de las citas bibliográficas y enunciados referidos; conocer los principios fundamentales de la redacción científica; escribir y revisar críticamente la escritura; lograr un uso apropiado del campus virtual de la UNER; y participar en instancias reales de discusión en torno a la escritura académica y científica.

\section{Los principales objetivos del curso fueron ofrecer un espacio para conocer algunos géneros académicos y científicos, sus partes y rasgos distintivos; lograr un manejo adecuado de las citas bibliográficas y enunciados referidos; conocer los princi- pios fundamentales de la redacción científica; escribir y revisar críticamente la escritura; lograr un uso apropiado del campus virtual de la UNER; y participar en instancias reales de discu- sión en torno a la escritura académica y científica.}

En este artículo se describe la propuesta del curso y la valoración de los participantes desde una intención de construir conocimiento sobre la práctica docente en entornos virtuales. En ese sentido, la investigación educativa pretende actuar directamente sobre los procesos de enseñanza y aprendizaje con la intención de mejorarlos (Blández, 2010).

\section{METODOLOGÍA}

Se presenta una investigación educativa en la que se describe una experiencia de virtualización de una unidad en formato curso virtual abierto. Se pretende conocer la utilidad del curso a partir de las valoraciones de los participantes.

Se propuso la realización de un curso virtual de redacción académica y científica para los estudiantes de segundo año de la Licenciatura en Nutrición de la FB-UNER. También se contó con la participación de estudiantes avanzados y docentes de dicha institución. El mismo se ofreció en la plataforma virtual Moodle de la UNER. 
La metodología fue modalidad virtual, e-learning asincrónica y autoaprendizaje con tutor. Una vez por semana, se habilitó un documento PDF de lectura obligatoria diseñado por la docente a cargo del curso. Con apoyo de dicho documento los estudiantes debieron realizar una tarea y participar en un foro temático. Las tareas consistían en reconocer tipologías y géneros, identificar las partes que conforman un trabajo académico o científico, construir una referencia bibliográfica siguiendo Normas APA, reconocer rasgos propios del discurso académico-científico, por mencionar algunos ejemplos. Se ofrecieron también lecturas complementarias. Al final del curso, debieron realizar un trabajo final integrador.

Tomando como antecedente una experiencia realizada en 2012 en los talleres de lectura y escritura para primer año de todas las carreras presenciales de la FB-UNER (Clerici, 2016), se revisó la misma a partir de la valoración de los participantes. En esa primera propuesta, los foros no solicitaban actividades colaborativas en las que fuese necesario llegar a acuerdos mediados por tecnologías sino más bien responder interrogantes y preguntas de reflexión sin la exigencia explícita de que hubiese intercambio, lo que llevó a una participación aislada de las intervenciones de los demás. Eso a su vez se reflejó en un cuestionamiento al sentido de los foros a la hora de la valoración de la experiencia. Por eso, en esta propuesta, las actividades en los foros solicitaban aporte a un hilo de discusión o trabajo en pequeños grupos que implicaban diálogo y consenso.

La alfabetización busca ampliar en las personas la capacidad de comunicarse con los demás. En esta línea, el trabajo en foros temáticos buscó colocar al estudiante en una situación real de comunicación y servir de herramienta de diálogo y trabajo colaborativo. Los foros fueron asincrónicos, para permitir una participación reflexiva, frente a otras herramientas de comunicación y trabajo de carácter sincrónico, donde la inmediatez supone un obstáculo a la reflexión y el análisis. En el foro los estudiantes cuentan con más tiempo para organizar y escribir las ideas propias, revisar la escritura, y reflexionar sobre las opiniones de los demás participantes.

La alfabetización busca ampliar en las personas la capacidad de comunicarse con los demás. En esta línea, el trabajo en foros temáticos buscó colocar al estudiante en una situación real de comunicación y servir de herramienta de diálogo y trabajo colaborativo. Los foros fueron asincrónicos, para permitir una participación reflexiva, frente a otras herramientas de comunicación y trabajo de carácter sincrónico, donde la inmediatez supone un obstáculo a la reflexión y el análisis. 
En cuanto al trabajo de los estudiantes de segundo año con estudiantes avanzados y docentes, el foro potenció la comunicación y colaboración que suponen una motivación derivada del hecho de ponerse en contacto con otros miembros de la comunidad universitaria. Además, la relación directa con otros estudiantes supuso un enriquecimiento personal. En los foros, la clave es la colaboración, entendida como lo hace Bereiter (2002) como un "discurso progresivo". En ese discurso progresivo en una comunidad de aprendizaje "los errores y las malas interpretaciones se ven como oportunidades de ir más allá. Los participantes negocian sus ideas con los otros. Cada estudiante es miembro de un equipo, de una comunidad de aprendizaje y, por tanto, produce conocimiento." (Gros Salvat, 2008, p. 81)

La puesta en marcha del curso estuvo a cargo de la docente de Metodología de la Investigación I de la Licenciatura en Nutrición y los foros de cada módulo fueron tutoreado por las auxiliares de cátedra. Se solicitaron dos aulas virtuales para un trabajo en grupos reducidos.

El curso se organizó en módulos, cada uno con contenidos específicos: Módulo 0: Reconocimiento del entorno virtual, actualización de perfil y presentación en foro. Módulo 1: Géneros académicos y científicos. Módulo 2: La estructura de los trabajos científicos. Módulo 3: La cita bibliográfica. Enunciados referidos. Normas APA. Módulo 4: Principios fundamentales de la redacción científica. La retórica científica.

Además de los 31 estudiantes de segundo año, se inscribieron 24 docentes y estudiantes avanzados, particularmente a miembros de equipos de investigación que participan en los Proyectos de Investigación desde las Cátedras de la FB-UNER. Luego del curso se aplicó una encuesta de satisfacción anónima en línea.

El curso se organizó en módulos, cada uno con contenidos específicos: Módulo 0: Reconocimiento del entorno virtual, actualización de perfil y presentación en foro. Módulo 1: Géneros académicos y científicos. Módulo 2: La estructura de los trabajos científicos. Módulo 3: La cita bibliográfica. Enunciados referidos. Normas APA. Módulo 4: Principios fundamentales de la redacción científica. La retórica científica.

Además de los 31 estudiantes de segundo año, se inscribieron 24 docentes y estudiantes avanzados, particularmente a miembros de equipos de investigación que participan en los Proyectos de Investigación desde las Cátedras de la FB-UNER. Luego del curso se aplicó una encuesta de satisfacción anónima en línea. 


\section{RESULTADOS}

De los 31 estudiantes de segundo año, completaron el curso un total de 24 (77,4\%). Muchos de los que no lograron terminar el curso fueron quienes habían abandonado la carrera o la asignatura hacia el final del semestre. De los 26 participantes externos, fueron 18 los que entraron al menos una vez al aula y, de ellos, 16 (88,8\%) completaron todos los requisitos de aprobación. Al contactar a quienes adeudaban tareas, para conocer los motivos por los cuales no habían podido completarlas, la totalidad lo justificó en la falta de tiempo.

En la encuesta de satisfacción se completaron un total de 26 formularios. Con la misma se buscó conocer la valoración general del curso, de los contenidos, de los materiales de lectura de cada módulo, de las actividades, de la consigna de trabajo final, del acompañamiento de la docente y la tutora y del propio desempeño como estudiante en el curso. Para cada pregunta tuvieron que elegir entre las opciones: excelente, muy bueno, bueno, regular y malo. Además, se les preguntó si lo recomendarían y qué sugerencias harían para mejorar la propuesta.

La valoración general del curso fue positiva ( $30,8 \%$ excelente, $57,7 \%$ muy bueno y 11, 5\% bueno). Entre los comentarios, expresaron: "Involucra varios aspectos de la temática pero no se limita. Abre debates sobre cuestiones inherentes a lo que ocurre en la realidad, en la práctica diaria, tanto desde el punto de vista de los docentes y alumnos". La valoración discriminada por aspectos se observa en la Figura 1.

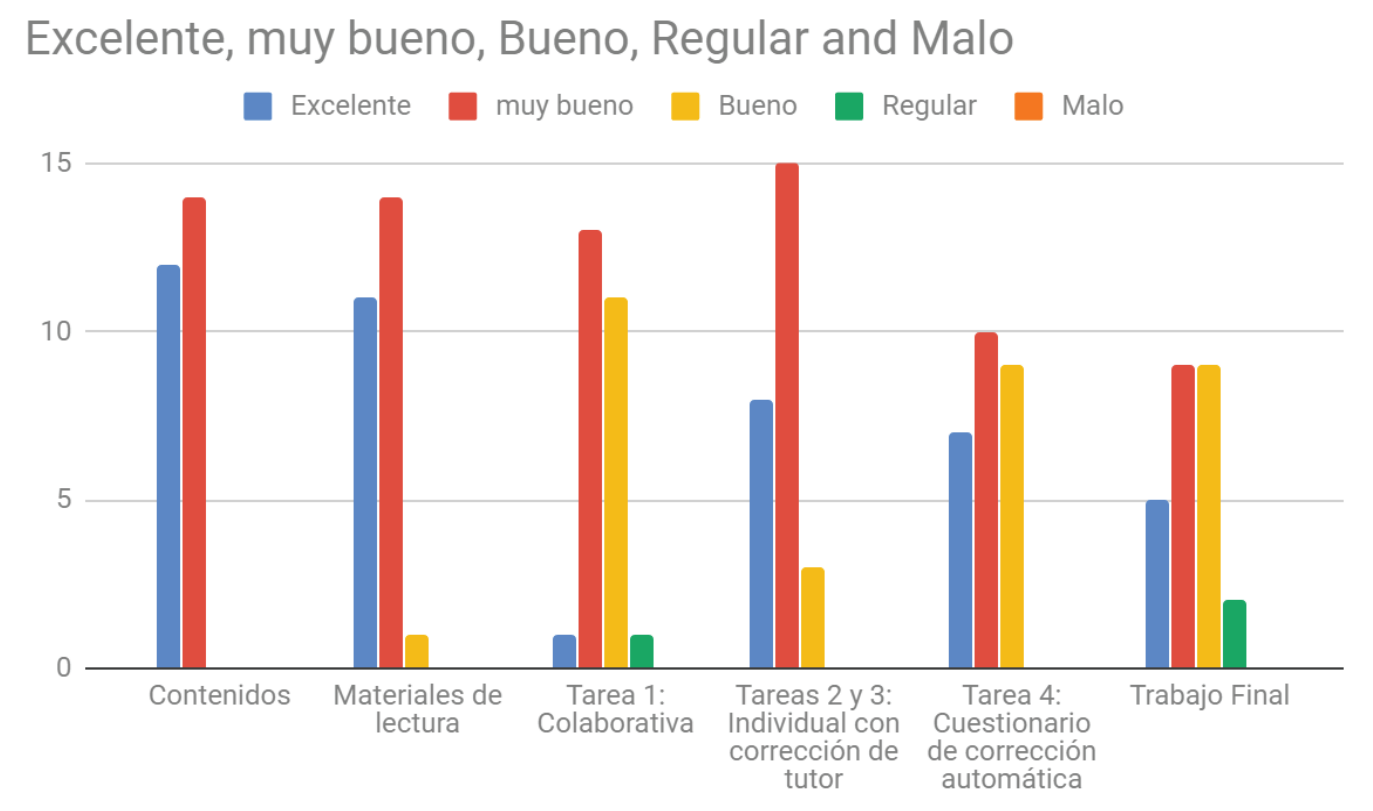

Figura 1. Valoración de aspectos del curso. 
Al momento de valorar los contenidos y los materiales de lectura expresaron que fueron útiles, completos, de fácil acceso, con lecturas complementarias que invitan a interiorizarse. Los consideraron claros, pertinentes y con ejemplos accesibles.

Las tareas presentaron diferentes niveles de complejidad que se reflejan en la valoración de los estudiantes. La tarea 1 consistió en una actividad de discusión que requería el logro de acuerdos utilizando un foro, las respuestas posibles para dicha tarea no estaban predeterminadas, sino que se construían colaborativamente. Las tareas 2 y 3 eran de resolución individual y se enviaban al tutor para la corrección. La tarea 4 fue un cuestionario de corrección automática e inmediata, que ofrecía una nota una vez terminado. De estas tareas, la que mostró una valoración menos favorable fue la 1, justificada por los participantes en la complejidad que implica trabajar en grupo en un entorno virtual, fundamentalmente, al comienzo del curso, cuando todavía no se conocían. Así lo expresaron:

Creo que esta tarea fue muy buena en cuanto a contenido y objetivos, pero considero debería haber sido la tarea número 2 o 3, ya que no nos conocíamos casi como grupo y la organización fue un poco difícil.

Cuesta realizarlas ya que no todos nos conectamos al mismo tiempo.

Era medio difícil intercambiar respuestas sin verse personalmente con los demás integrantes.

Me gustó el desafío de realizar una tarea grupal, sin embargo reconozco que no fue sencillo. Sugiero que la tarea grupal no sea la primera del curso, así se da tiempo a que los/las participantes nos conozcamos un poquito más!

La consigna del trabajo final ofrecía tres opciones semejantes a las realizadas durante el curso. La valoración osciló entre quienes expresaron que no se sentían preparados para realizarla y quienes destacaron como positivo la extensión, la posibilidad de integrar contenidos y de elegir entre las opciones propuestas. Algunos de sus testimonios así lo reflejan:

Aquí destaco por sobre todo la extensión del trabajo. Con 1 o 2 páginas se puede aplicar correctamente todos los contenidos aprendidos en el curso; no necesariamente tiene que ser un trabajo extenso por ser un trabajo final. Destacó la planificación de esta actividad por parte de las docentes a cargo del curso.

Me costó ya que no me sentía capaz para realizarla.

Me gustó mucho que existan tres propuestas para realizar el trabajo final.

La valoración del acompañamiento de la docente y las tutoras fue positiva (69,2\% excelente, $26,9 \%$ muy bueno y $3,8 \%$ bueno). Entre los comentarios que dejaron en el formulario se destacan: 
Había una comunicación fluida y siempre que necesitaba ayuda me la brindaba al instante

La participación en la moderación de foros, las observaciones, la motivación siempre estuvieron presentes. Me gustó la idea de que a partir de ciertas inquietudes se fomentará el debate.

La valoración del propio desempeño como estudiantes fue menos favorable $(7,7 \%$ excelente, $23,1 \%$ muy bueno, $53,8 \%$ bueno y $15,4 \%$ regular). No obstante, muchos destacaron que lograron sostener el esfuerzo para completar las tareas. Entre los comentarios, expresaron que:

Me fue difícil organizarme en conjunto con mis compañeros para la primera actividad, de igual manera llegamos a entregarla en la fecha establecida. Luego creo tuve buen desempeño, tomándome el tiempo necesario para leer y releer los materiales de lectura que se proponían en cada módulo y luego realizando la tarea con responsabilidad y compromiso. Participe en foros de intercambio con mis compañeros, leyendo sus comentarios y respondiendo algunos. Entregué tareas en tiempo y forma. Reconozco que no puse suficiente empeño pero llegué a realizar todas las actividades.

Me parece un tema muy importante, al que debemos tiempo de práctica. Por mi parte me esforcé por cumplir con las lecturas y trabajos a conciencia, porque realmente me es útil

La totalidad afirma que recomendaría el curso por la utilidad de los contenidos y por el acompañamiento de la docente y las tutoras. En cuanto a los contenidos, destacaron que la alfabetización académica debe ser parte de la formación. Algunos destacaron la modalidad virtual como novedosa. Se destacan algunos de sus comentarios:

Es muy útil para el desenvolvimiento académico cuando se nos exigen trabajos de informes y monográficos, y al final para la tesis. Son herramientas que vamos a utilizar siempre.

Principalmente por su didáctica. Es sencillo, "fácil de engancharse y de seguirlo" pero con un contenido muy bueno y enriquecedor.

Porque te brinda información que muchas veces no se tiene. Por eso creo conveniente recomendarla.

Porque son temas interesantes y sumamente necesarios tenerlos presentes, tanto para elaborar un informe cómo escribir un Trabajo Final Integrador o Tesina.

Porque ayuda a conocer más profundamente sobre normas APA que es muy práctica su utilización.

Por la dinámica, es práctico, los temas abordados son parte de las actividades diarias, promueve las actividades colaborativas e intercambio con otros. 
Finalmente, se solicitó a los participantes que expresaran qué mejoras sugerirían para esta propuesta. En general se relacionan con los plazos de entrega, la complejidad y cantidad de tareas, la modalidad virtual y la conformación de los grupos.

\section{DISCUSIÓN}

Los participantes consideran que la experiencia les permitió aprender y rescatan principalmente cuestiones vinculadas con la escritura. El nivel de satisfacción en general fue elevado, aún en el caso de los estudiantes de segundo año, para quienes se trató de una actividad obligatoria. Las principales dificultades encontradas se relacionan con la falta de tiempo y el bajo aprovechamiento de la interacción en foros como un espacio de aprendizaje colaborativo. Hay aquí un camino por recorrer.

Según algunos autores, la utilización de foros virtuales genera cierta renuencia. Pérez Pérez (2004) señala entre dichos motivos la resistencia al esfuerzo; dificultades para la expresión escrita; dificultades para la lectura; timidez ante la opinión de los compañeros; resistencia al trabajo sistemático que introduzca pautas y límites temporales; falta de autonomía y criterios propios para participar; disponibilidad de tiempo.

Con respecto a la actividad colaborativa, esa tarea fue propuesta como mejora a la primera edición y, si bien resultó una instancia enriquecedora, no fue acertado ubicarla al inicio del curso. Para futuras ediciones de la propuesta, se plantea la realización de la tarea grupal colaborativa en el último módulo para dar tiempo al afianzamiento en el trabajo en entornos virtuales.

A juzgar por los resultados de este trabajo, se puede concluir que la virtualización de una unidad en formato curso virtual abierto ha permitido ofrecer a los estudiantes una experiencia genuina de intercambio con la comunidad educativa. El curso significó una oportunidad de interacción entre estudiantes y el resto de la comunidad universitaria en torno a una instancia de formación como es un curso de redacción científica. 


\section{BIBLIOGRAFÍA}

Arnoux, Elvira; Di Stefano, Mariana \& Pereira, Cecilia. (2002). “La lectura y la escritura en la universidad". Buenos Aires: Eudeba.

Bereiter, Carl. (2002). "Design research for sustained innovation: Cognitive Studies Bulletin of the Japanese Cognitive Science Society", pp. 321-327. En: B. Gros Salvat (2008) Aprendizajes, conexiones y artefactos. la producción colaborativa del conocimiento. Barcelona: Gedisa.

Blández, Julia. (2010). “La clase de educación física: escenario de la investigación". En C. González y T. Lleixà (coords.), Educación Física. Investigación, innovación y buenas prácticas (pp. 43-58). Barcelona: Graó.

Carlino, Paula. (2003a). “Alfabetización Académica: Un cambio necesario, algunas alternativas posible". Educere. Investigación, vol. 6, núm. 20, pp. 409-420.

Carlino, Paula. (2003b). “Leer textos científicos y académicos en la educación superior: Obstáculos y bienvenida a una cultura nueva". Uni-pluri/versidad, vol. 3, núm. 2, pp. 17-23.

Clerici, Carolina (2016). “Curso en línea de redacción científica como espacio de aprendizaje e intercambio entre ingresantes y el resto de la comunidad universitaria". Diálogos Pedagógicos, vol. 14, núm. 28, pp. 142-149.

Clerici, Carolina; Monteverde, Ana Clara \& Fernández, Andrea (2015). “Lectura, escritura y rendimiento académico en ingresantes universitarios". Ciencia, Docencia y Tecnología, vol. 26, núm. 50, 35-70.Recuperado de http://pcient. uner.edu.ar/index.php/cdyt/article/view/59

Clerici, Carolina; Truffer, Isabel \& Unamuzaga, María Elena (2009, agosto 6-8). "Dificultades y habilidades para la lectura y la escritura (LyE) en el ingresante". V Jornadas Internacional de Educación Lingüística, Concordia, Argentina.

González de la Torre, Yolanda; Jiménez Mora, José \& Rosas, Jorge Ignacio. (2016). "Prácticas lectoras de estudiantes universitarios con fines de escritura académica". Actualidades Investigativas en Educación, vol. 16, núm. 1, pp. 233252. doi: 10.15517/aie.v16i1.21971

Gros Salvat, Begoña (2008). “Aprendizajes, conexiones y artefactos. La producción colaborativa del conocimiento". Barcelona: Gedisa

Pérez Pérez, Ramón (2004). "Mida las tareas de aprendizaje". En: L. M. Villar Angulo (coord.) Programa para la mejora de la docencia universitaria (pp. 523542). Madrid: Pearson-Prentice Hall. 
Rogoff, Barbara. (1997). "Los tres planos de la actividad sociocultural: apropiación participativa, participación guiada y aprendizaje". En: J. Wertsch, P, del Río y A. Álvarez (Eds.) La mente sociocultural. Aproximaciones teóricas y aplicadas (pp.111-128). Madrid: Fundación Infancia y Aprendizaje.

Vigotsky, Lev. (1987). "El desarrollo de los procesos psicológicos superiores". Madrid: Crítica Grijalbo. 